\title{
Green's function for a spherical dielectric discontinuity and its application to simulation
}

\author{
Per Linse ${ }^{1, \text { a) }}$ and Leo Lue Le $^{2, b}$ \\ ${ }^{1)}$ Physical Chemistry, Department of Chemistry \\ Lund University, P.O. Box 124, S-221 00 Lund, Sweden \\ ${ }^{2)}$ Department of Chemical and Process Engineering, University of Strathclyde \\ James Weir Building, 75 Montrose Street, Glasgow G1 1XJ, \\ United Kingdom
}

(Dated: 31 December 2013)

We present rapidly convergent expressions for the Green's function of the Poisson equation for spherically symmetric systems where the dielectric constant varies discontinuously in the radial direction. These expressions are used in Monte Carlo simulations of various electrolyte systems, and their efficiency is assessed. With only the leading term of the expansion included, a precision of the polarization energy of $0.01 \mathrm{~kJ} \mathrm{~mol}^{-1}$ or better was achieved, which is smaller than the statistical uncertainty of a typical simulation. The inclusion of the dielectric inhomogeneity leads to a 2.5 -fold increase of the computational effort, which is modest for this type of model. The simulations are performed on six types of systems having either (i) a uniform surface charge distribution, (ii) a uniform volume charge distribution, or (iii) mobile ions, which were neutralized by mobile counterions. The ion density distributions are investigated for different dielectric conditions. These spatial distributions are discussed in terms of the importance of (i) the direct mean-field Coulomb interaction, (ii) the surface charge polarization at the dielectric discontinuity, and/or (iii) the change in the attractive Coulomb correlations.

\footnotetext{
a)Electronic mail: per.linse@ @ fkem1.lu.se

b)Electronic mail: leo.lue@strath.ac.uk
} 


\section{INTRODUCTION}

Soft matter is a widespread field containing colloid particles, proteins, polymers, phospholipids, surfactants, etc and their organization in solution. Properties of soft matter containing charged species are often strongly affected by Coulomb interactions. ${ }^{1,2}$ The primitive model of electrolytes is often used to describe systems containing Coulomb interactions. Within this model, the charges are explicitly included and the solvent is represented by a dielectric medium that attenuates the Coulomb interactions. The primitive model implies a nonphysical uniform dielectric constant throughout the whole system, including the interior of the low-dielectric species.

The spatial dependence of the electrical potential follows the Poisson equation. ${ }^{3,4}$ As a consequence, the electric potential from a charge near a single and planar dielectric discontinuity can be represented by a single imaginary (image) charge located on the other side of the discontinuity where the potential is evaluated. However, for curved dielectric discontinuities, the expression of the reaction potential is more complex, and it cannot, in general, be expressed as generated by a finite set of image charges. Hence, for curved discontinuities, we have infinite expansions that can not be analytically summed to yield a simple expression, such as that for the planar case.

Attempts at improving the primitive model by allowing nonuniform dielectric properties of models with spherical dielectric discontinuities stems back to, among others, Tanford and Kirkwood. ${ }^{5}$ They derived relevant equations, provided expressions applicable for large differences in the dielectric constants, and applied those onto globular proteins. Later Torrie and Valleau ${ }^{6}$ and Bratko, Jönsson, and Wennerström, ${ }^{7}$ included the reaction potential from the surface polarization appearing at planar dielectric discontinuities in Monte Carlo (MC) simulations. Linse ${ }^{8}$ applied a similar, but approximate, MC approach to spherical dielectric discontinuities covering mobile charges located both inside and outside the discontinuity. Early investigations of planar dielectric discontinuities with mobile ions have also been performed by Levine and Outhwaite within the framework of the Poisson-Boltzmann equation ${ }^{9}$ and Kjellander and Marcelja within the hypernetted chain approximation. ${ }^{10}$

More recently, a renewed interest has appeared to describe the electrical potential outside a spherical dielectric discontinuity generated by charges located outside the discontinuity. Messina ${ }^{11}$ provided a faster scheme of summing the infinite expansion appearing in a spherical geometry. A further step on this issue was taken by dos Santos et al., ${ }^{12}$ which was based on the fact that the reaction potential could be represented by the potential of an image charge and a nonuniform line 
charge inside the sphere. ${ }^{13}$ Later, Lue and Linse ${ }^{14}$ investigated and compared three routes to sum the polarization energy. In general, the fastest route involved a reformulation of a factor containing the ratios of the two dielectric constants and a summation index into a very rapidly convergent sum. The simulation time of including a single dielectric surface was here reduced down to only a factor two to three times that of a homogeneous dielectric medium.

The methods proposed by Lue and Linse ${ }^{14}$ provided the potential outside the spherical dielectric discontinuity generated by the surface polarization caused by a charge outside the discontinuity. In the present contribution, we extend our method to the four combinations of having the field point and the source location inside/outside the sphere. In particular, we demonstrate that the same numerical advantages of reformulating the infinite expression to a much faster sum prevails in all four cases. We find that the simulation time increases only 2.5 times that of a homogeneous dielectric medium to obtain a convergence similar or better than the statistical uncertainty of extended MC simulations. Thus, with this approach, basically all systems containing a spherical dielectric discontinuity can be simulated with an efficiency not differing much from that of the corresponding homogeneous dielectric system.

In this work, we also explore the behavior of these model systems containing a uniform surface charge distribution, a uniform volume charge distribution, or mobile ions, in each case neutralized by mobile counterions. We consider cases where ions are restricted to one side of the dielectric discontinuity and where they occupy a volume extending on both sides of the dielectric discontinuity.

The remainder of this paper is organized as follows. First, rapidly convergent expressions for the Green's function of a system with a single spherical dielectric discontinuity in a background continuum solvent are presented in Sec. II. A physical interpretation for these expressions is also given in that section. The model, the simulation method, and the parameters of the systems used to examine the rapidly convergent expressions for the Green's function are provided in Sec. III. The numerical results and their discussion are given in Sec. IV. Finally, the work is summarized in Sec. V. 


\section{GREEN'S FUNCTION FOR A SINGLE SPHERICAL DIELECTRIC DISCONTINUITY}

In this section, we develop expressions for the Green's function of the Poisson equation applicable to a three-dimensional space containing a spherical dielectric discontinuity. Physically, the Green's function $G_{0}\left(\mathbf{r}, \mathbf{r}^{\prime}\right)$ represents the potential generated at position $\mathbf{r}$ by a unit point charge located at position $\mathbf{r}^{\prime}$ and is defined through the equation

$$
-\frac{1}{4 \pi} \nabla \cdot\left[\varepsilon(\mathbf{r}) \nabla G_{0}\left(\mathbf{r}, \mathbf{r}^{\prime}\right)\right]=\delta^{d}\left(\mathbf{r}-\mathbf{r}^{\prime}\right)
$$

where $\varepsilon(\mathbf{r})$ denotes the spatially variation of the dielectric constant, and $d$ the dimensionality of the space.

Throughout, we consider a three-dimensional space $(d=3)$ containing a system composed of a sphere of radius $R$ with a dielectric constant of $\varepsilon_{1}$ embedded in a medium with a dielectric constant $\varepsilon_{2}$. At these conditions, the Green's function for the Poisson equation can be written as

$$
G_{0}\left(\mathbf{r}, \mathbf{r}^{\prime}\right)=\frac{1}{\varepsilon_{2}\left|\mathbf{r}-\mathbf{r}^{\prime}\right|}+\delta G_{0}\left(\mathbf{r}, \mathbf{r}^{\prime}\right)
$$

where the first term in Eq. (2) is the Green's function for a bulk system with the dielectric constant $\varepsilon_{2}$, and $\delta G_{0}$ is the contribution due to any inhomogeneities in the dielectric constant, i.e. the polarization contribution. Furthermore, $\delta G_{0}$ can be expressed as ${ }^{4,15}$

$$
\delta G_{0}\left(\mathbf{r}, \mathbf{r}^{\prime}\right)=\sum_{l=0}^{\infty} \sum_{m=-l}^{l} \delta g_{l}\left(r, r^{\prime}\right) Y_{l m}(\theta, \phi) Y_{l m}^{*}\left(\theta^{\prime}, \phi^{\prime}\right)
$$

where

$$
\delta g_{l}\left(r, r^{\prime}\right)=\frac{4 \pi}{2 l+1} \frac{1}{\varepsilon_{2} R} \begin{cases}-\frac{\Delta}{\eta} \frac{(l+1)}{l+\zeta}\left(\frac{r}{R} \frac{r^{\prime}}{R}\right)^{l} & \\ +\left(\eta^{-1}-1\right)\left(\frac{r_{<}}{R}\right)^{l}\left(\frac{R}{r_{>}}\right)^{l+1} & \text { for } r, r^{\prime}<R \\ \frac{\Delta l}{l+\zeta}\left(\frac{r_{<}}{R}\right)^{l}\left(\frac{R}{r_{>}}\right)^{l+1} & \text { for } r<R \text { and } r^{\prime}>R \\ \frac{\Delta l}{l+\zeta}\left(\frac{R}{r} \frac{R}{r^{\prime}}\right)^{l+1} & \text { for } r, r^{\prime}>R\end{cases}
$$


with

$$
\begin{aligned}
\eta & \equiv \varepsilon_{1} / \varepsilon_{2} \\
\Delta & \equiv(1-\eta) /(1+\eta) \\
\zeta & \equiv(1+\eta)^{-1} \\
r_{<} & \equiv \min \left(r, r^{\prime}\right) \quad r_{>} \equiv \max \left(r, r^{\prime}\right),
\end{aligned}
$$

and where $r \equiv|\mathbf{r}|$, and $Y_{l m}(\theta, \phi)$ denotes the spherical harmonics.

A list of important variables and their symbols is provided in Appendix A.

\section{A. Physical interpretation}

Consider the potential at a position $\mathbf{r}$, located inside the dielectric sphere, generated by a unit point charge located at a position $\mathbf{r}^{\prime}$. This potential is composed of a direct contribution due to the unit point charge and an indirect contribution due to the polarization of the dielectric interface by the point charge. The potential due to the polarization can physically be represented by a point charge located at the Kelvin point $\mathbf{r}_{\mathrm{K}}$, which lies outside the sphere, and a line charge with a nonuniform charge density, which stretches from the Kelvin point to infinity. This is schematically depicted in Figs. 1(a) and (c). In this case, the Green's function can be written as

$$
G_{0}\left(\mathbf{r}, \mathbf{r}^{\prime}\right)=\frac{1}{\varepsilon_{1}\left|\mathbf{r}-\mathbf{r}^{\prime}\right|}+\frac{q_{\mathrm{K}}}{\varepsilon_{1}\left|\mathbf{r}-\mathbf{r}_{\mathrm{K}}\right|}+\frac{1}{\varepsilon_{1}} \int_{r_{\mathrm{K}}}^{\infty} d x \frac{\lambda_{\mathrm{K}}(x)}{|\mathbf{r}-\mathbf{x}|}
$$

The first term is the direct potential generated by the unit point charge, the second term is the potential from the point charge located at the Kelvin point, and the final term is the potential from the line charge.

The location $\mathbf{r}_{\mathrm{K}}$ of the Kelvin point depends on the position $\mathbf{r}^{\prime}$ of the unit point charge as

$$
\mathbf{r}_{\mathrm{K}}=\mathbf{r}^{\prime} \begin{cases}\left(R / r^{\prime}\right)^{2} & \text { for } r^{\prime}<R \\ 1 & \text { for } R<r^{\prime}\end{cases}
$$

Note, when the unit point charge is outside the sphere, $\mathbf{r}_{\mathrm{K}}$ is coincident with the location of the point charge [see Fig. 1 (c)]. Also, the magnitude $q_{\mathrm{K}}$ of the point charge at the Kelvin point 
depends on the position of the unit point charge according to

$$
q_{\mathrm{K}}=-\Delta\left\{\begin{array}{ll}
r_{\mathrm{K}} / R & \text { for } r^{\prime}<R \\
1 & \text { for } R<r^{\prime}
\end{array} .\right.
$$

The charge density of the line charge is given by

$$
\lambda_{\mathrm{K}}(x)=-\Delta \zeta \eta\left(\frac{r_{\mathrm{K}}}{x}\right)^{\zeta}\left\{\begin{array}{l}
R^{-1} \text { for } r^{\prime}<R \\
r_{\mathrm{K}}^{-1} \text { for } R<r^{\prime}
\end{array}\right.
$$

where $x$ is the distance from the center of the dielectric sphere. Note that the sign of the line charge is the same to that of the point charge at the Kelvin point.

Now, we consider the potential at $\mathbf{r}$ outside the dielectric sphere generated by a unit point charge located at $\mathbf{r}^{\prime}$. In this case, the potential due to the polarization of the dielectric interface can also be represented by a point charge located at the Kelvin point $\mathbf{r}_{\mathrm{K}}$ now lying inside the sphere and a line charge stretching from the Kelvin point to the center of the dielectric sphere. This is depicted in Figs. 1(b) and (d). The Green's function can be written in a form very similar to that of Eq. (9) according to

$$
G_{0}\left(\mathbf{r}, \mathbf{r}^{\prime}\right)=\frac{1}{\varepsilon_{2}\left|\mathbf{r}-\mathbf{r}^{\prime}\right|}+\frac{q_{\mathrm{K}}}{\varepsilon_{2}\left|\mathbf{r}-\mathbf{r}_{\mathrm{K}}\right|}+\frac{1}{\varepsilon_{2}} \int_{0}^{r_{\mathrm{K}}} d x \frac{\lambda_{\mathrm{K}}(x)}{|\mathbf{r}-\mathbf{x}|}
$$

The location of the Kelvin point is given by

$$
\mathbf{r}_{\mathrm{K}}=\mathbf{r}^{\prime}\left\{\begin{array}{ll}
1 & \text { for } r^{\prime}<R \\
\left(R / r^{\prime}\right)^{2} & \text { for } R<r^{\prime}
\end{array},\right.
$$

and the magnitude $q_{\mathrm{K}}$ of the point charge at the Kelvin point becomes

$$
q_{\mathrm{K}}=\Delta \begin{cases}1 & \text { for } r^{\prime}<R \\ r_{\mathrm{K}} / R & \text { for } R<r^{\prime}\end{cases}
$$

The charge density of the line charge is given by

$$
\lambda_{\mathrm{K}}(x)=-\Delta \zeta\left(\frac{r_{\mathrm{K}}}{x}\right)^{1-\zeta}\left\{\begin{array}{l}
r_{\mathrm{K}}^{-1} \text { for } r^{\prime}<R \\
R^{-1} \text { for } R<r^{\prime}
\end{array}\right.
$$


Note that in this case, the line charge density has the opposite sign as that of the Kelvin point charge.

\section{B. Rapidly convergent expressions}

The Green's function can be written in a more rapidly convergent series. ${ }^{14}$ Here, we need to split into three different cases depending on the locations of the point $\mathbf{r}$, at which the potential should be expressed, and of the point charge $\mathbf{r}^{\prime}$. See Appendix B for derivations of the expressions in this subsection.

Case 1: $r<R$ and $r^{\prime}<R$

For the case where both $\mathbf{r}$ and $\mathbf{r}^{\prime}$ lie inside the dielectric discontinuity [Fig. 1(a)], the Green's function can be written as

$$
\begin{gathered}
\delta G_{0}\left(\mathbf{r}, \mathbf{r}^{\prime}\right)=\frac{\eta^{-1}-1}{\varepsilon_{2}\left|\mathbf{r}-\mathbf{r}^{\prime}\right|}+\frac{\eta}{\varepsilon_{1} R} \frac{1-\eta}{1+\eta}\left\{-\frac{1}{\eta}\left(1-2 t \cos \gamma+t^{2}\right)^{-1 / 2}\right. \\
+\frac{t^{-1}}{1+\eta} \ln \frac{\left(1-2 t \cos \gamma+t^{2}\right)^{1 / 2}-t+\cos \gamma}{1+\cos \gamma} \\
\left.-\frac{\eta}{1+\eta}\left[1+\sum_{l=1}^{\infty} \frac{1}{(l+1)} \frac{t^{l} P_{l}(\cos \gamma)}{(1+\eta) l+1}\right]\right\}
\end{gathered}
$$

where $\gamma$ is the angle between vectors $\mathbf{r}$ and $\mathbf{r}^{\prime}$, and $t=r r^{\prime} / R^{2}$. The first term in the braces is the potential generated by a point charge of magnitude $q_{\mathrm{K}}=-\Delta r_{\mathrm{K}} / R$ that is located at position $\mathbf{r}_{\mathrm{K}}$. The second term in the braces represents the potential generated by a line charge of density $\lambda(x)=$ $-\Delta \zeta \eta r_{\mathrm{K}} /(x R)=-\Delta \zeta \eta R /\left(x r^{\prime}\right)$, where $x$ is the distance from the center of the dielectric sphere. The third term corresponds to a spherical surface charge distribution of total charge $-\Delta \eta \zeta r_{\mathrm{K}} / R$ and of radius $r_{\mathrm{K}}$. 
The corresponding expression for the total Green's function in this case is

$$
\begin{aligned}
G_{0}\left(\mathbf{r}, \mathbf{r}^{\prime}\right)= & \frac{1}{\varepsilon_{1}\left|\mathbf{r}-\mathbf{r}^{\prime}\right|}+\frac{\eta}{\varepsilon_{1} R} \frac{1-\eta}{1+\eta}\left\{-\frac{1}{\eta}\left(1-2 t \cos \gamma+t^{2}\right)^{-1 / 2}\right. \\
& +\frac{t^{-1}}{1+\eta} \ln \frac{\left(1-2 t \cos \gamma+t^{2}\right)^{1 / 2}-t+\cos \gamma}{1+\cos \gamma} \\
& \left.-\frac{\eta}{1+\eta}\left[1+\sum_{l=1}^{\infty} \frac{1}{(l+1)} \frac{t^{l} P_{l}(\cos \gamma)}{(1+\eta) l+1}\right]\right\}
\end{aligned}
$$

Case 2: $r_{<}<R$ and $r_{>}>R$

For the case where one of $\mathbf{r}$ and $\mathbf{r}^{\prime}$ lies inside and the other outside the dielectric discontinuity [Figs. 1(b) and (c)], we have

$$
\begin{aligned}
\delta G_{0}\left(\mathbf{r}, \mathbf{r}^{\prime}\right)=\frac{1}{\varepsilon_{2} r_{>}} & \frac{1-\eta}{1+\eta}\left\{\left(1-2 t \cos \gamma+t^{2}\right)^{-1 / 2}\right. \\
& +\frac{t^{-1}}{1+\eta} \ln \frac{\left(1-2 t \cos \gamma+t^{2}\right)^{1 / 2}-t+\cos \gamma}{1+\cos \gamma} \\
& \left.-\frac{\eta}{1+\eta}\left[1+\sum_{l=1}^{\infty} \frac{1}{l+1} \frac{t^{l} P_{l}(\cos \gamma)}{(1+\eta) l+1}\right]\right\}
\end{aligned}
$$

where $t=r_{<} / r_{>}$.

In the situation where the unit point charge is located inside the dielectric sphere [Fig. 1(b)], the first term corresponds to the potential generated by a point charge of magnitude $q_{\mathrm{K}}=\Delta$ located at the Kelvin point, while the second term corresponds to the potential generated by a line charge of uniform charge density $\lambda=-\Delta \zeta / r^{\prime}$, which extends from the center of the sphere to the point $\mathbf{r}^{\prime}$. The third term corresponds to a spherical surface charge distribution of total charge $-\Delta \eta \zeta$ and of radius $r_{\mathrm{K}}$.

In the case where the unit point charge is outside the dielectric sphere [Fig. 1(c)], the first term corresponds to a point charge of magnitude of $q_{\mathrm{K}}=-\Delta$ that is located at $\mathbf{r}^{\prime}$, and the second term represents the potential generated by a line charge of density $\lambda(x)=-\Delta \zeta \eta / x$, where $x$ is the distance from the center of the dielectric sphere, that extends from $\mathbf{r}^{\prime}$ to infinity. The third term corresponds to a spherical surface charge distribution of total charge $-\Delta \eta \zeta$ and of radius $r_{\mathrm{K}}$. 
Case 3: $r>R$ and $r^{\prime}>R$

For the case where both $\mathbf{r}$ and $\mathbf{r}^{\prime}$ lie outside the dielectric discontinuity [Figs. 1(d)], we get

$$
\begin{aligned}
\delta G_{0}\left(\mathbf{r}, \mathbf{r}^{\prime}\right)=\frac{1}{\varepsilon_{2} R} & \frac{1-\eta}{1+\eta}\left\{t\left(1-2 t \cos \gamma+t^{2}\right)^{-1 / 2}\right. \\
& +\frac{1}{1+\eta} \ln \frac{\left(1-2 t \cos \gamma+t^{2}\right)^{1 / 2}-t+\cos \gamma}{1+\cos \gamma} \\
& \left.-\frac{\eta t}{1+\eta}\left[1+\sum_{l=1}^{\infty} \frac{1}{l+1} \frac{t^{l} P_{l}(\cos \gamma)}{(1+\eta) l+1}\right]\right\}
\end{aligned}
$$

where $\gamma$ is the angle between vectors $\mathbf{r}$ and $\mathbf{r}^{\prime}$, and $t=R^{2} /\left(r r^{\prime}\right)$. The first term is from a point charge of magnitude $q_{\mathrm{K}}=\Delta r_{\mathrm{K}} / R=\Delta R / r^{\prime}$ that is located at the Kelvin point $\mathbf{r}_{\mathrm{K}}$. The second term is from a line charge with a uniform charge density $\lambda=-\Delta \zeta / R$ stretching from the center of the dielectric sphere to the Kelvin point. The third term is from a spherical charge distribution of total charge $-\Delta \eta \zeta r_{\mathrm{K}} / R$ and of radius $r_{\mathrm{K}}$.

\section{MODEL AND METHODS}

In this section, we will describe the model, methods, and systems containing a spherical dielectric discontinuity, used in our study of (i) the convergence of expressions given by Eqs. (17), (19), and (20) and (ii) how structural properties of these systems depends on the dielectric inhomogeneities.

\section{A. Model}

Throughout this work, all systems possess a spherical region, which is labeled 1, with a radius $R_{1}$, area $S_{1}=4 \pi R_{1}^{2}$, volume $V_{1}=4 \pi R_{1}^{3} / 3$, and dielectric constant $\varepsilon_{1}$ (see Fig. 2). Region 1 is immersed in medium 2, which is of infinite extension and has the dielectric constant $\varepsilon_{2}$. Hence, when $\varepsilon_{2} \neq \varepsilon_{1}$, we have a spherical dielectric discontinuity at $R_{1}$. Some systems contain a second sphere with radius $R_{2}>R_{1}$, placed concentrically around region 1, and hence sphere 2 divides medium 2 into two parts. The space between spheres 1 and 2 is referred as to region 2, which consequently has the volume $V_{2}=4 \pi R_{2}^{3} / 3-V_{1}$.

In the model, charge appears as mobile ions and stationary charge densities. Ions are present in 
all systems and appear as charged hard spheres of radius $R_{\text {ion }}=2 \AA$ and charge $q=z_{\text {ion }} e_{0}$, where $e_{0}$ is the fundamental unit of charge.

Beside mobile ions, some systems also have a stationary charge density. Such a density generates a potential that acts on the ions, and this potential is referred to as an "external potential". Two such stationary charge distributions will be employed.

The first one involves an infinite thin shell of a uniform surface charge density $\sigma(\mathbf{r})$ at the interface between region 1 and 2 and given by

$$
\sigma(\mathbf{r})=\frac{Q_{S}}{S_{1}} \delta\left(r-R_{1}\right)
$$

where $Q_{S}$ is the total charge of the spherical shell. When region 1 is immersed in a continuum medium with the dielectric constant $\varepsilon_{2}$, the electrostatic potential at $\mathbf{r}$ generated by the surface charge density $\sigma\left(\mathbf{r}^{\prime}\right)$ becomes

$$
\int d \mathbf{r}^{\prime} G_{0}\left(\mathbf{r}, \mathbf{r}^{\prime}\right) \sigma\left(\mathbf{r}^{\prime}\right)=\frac{Q_{S}}{\varepsilon_{2} R_{1}} \begin{cases}1 & \text { for } r<R_{1} \\ R_{1} / r & \text { for } R_{1}<r\end{cases}
$$

Ions within region 1 experience a constant electrostatic potential from the surface charge density, regardless of their position within the region. In region 2, ions will experience an electrostatic potential that decays inversely with their distance from the center of region 1.

The second stationary charge distribution is a uniform volume charge density $\rho(\mathbf{r})$ in region 1 , given by

$$
\rho(\mathbf{r})=\frac{Q_{V}}{V_{1}}\left\{\begin{array}{l}
1 \text { for } r<R_{1} \\
0 \text { for } R_{1}<r
\end{array}\right.
$$

where $r$ is the distance from the center of region 1 , and $Q_{V}$ is the total charge of region 1 . When region 1 is immersed in a medium with dielectric constant $\varepsilon_{2}$, the electrostatic potential generated at $\mathbf{r}$ by the volume charge density $\rho\left(\mathbf{r}^{\prime}\right)$ becomes

$$
\int d \mathbf{r}^{\prime} G_{0}\left(\mathbf{r}, \mathbf{r}^{\prime}\right) \rho\left(\mathbf{r}^{\prime}\right)=\frac{Q_{V}}{\varepsilon_{1} R_{1}}\left\{\begin{array}{lc}
\frac{1}{2}\left(3-\frac{r^{2}}{R_{1}^{2}}\right)-(1-\eta) & \text { for } r<R_{1} \\
\frac{\eta R_{1}}{r} & \text { for } R_{1}<r
\end{array}\right.
$$

with $\eta \equiv \varepsilon_{1} / \varepsilon_{2}$. This external potential gives rise to a harmonic potential that brings ions toward the center of region 1 . 


\section{B. Method}

Metropolis Monte Carlo (MC) simulations in the canonical ensemble (i.e., at constant number of particles, volume, and temperature) are used to examine the different model systems. The potential energy of a system is composed of direct Coulomb and polarization terms. The polarization energy was evaluated using Eqs. (17), (19), and (20) with the upper infinite limit of their sums over $l$ replaced by $l_{\max }$. Moreover, no significant differences were observed between $l_{\max }=0$ and 1 (see Sec. IV A 1). Unless otherwise stated, data using $l_{\max }=0$ are presented. All energies are given in $\mathrm{kJ}$ (mol ions) $)^{-1}$.

After equilibration, each simulation involved $10^{6} \mathrm{MC}$ passes (trial moves per ion) for systems containing $N=100$ counterions and $10^{7} \mathrm{MC}$ passes for systems with $N<100$. The value of

trial displacements ranged from 2 to $10 \AA$. Radial ion density distributions were determined by using a histogram width of $0.2 \AA$. In passing, the statistical uncertainty of the potential energy and radial density distributions is, for normal requirements, unnecessarily small $-10^{4}$ passes would be sufficient in most cases.

The statistical uncertainties of potential energies were evaluated by repetitively dividing a simulation into blocks of an increasing number of configurations, evaluating the statistical uncertainty of each block length, and finally extrapolate to infinite block length. This protocol removes the influence of the statistical correlation between successive configuration inherent in the Metropolis Monte Carlo procedure. Uncertainties of radial density distributions were obtained by the simpler method of divining a run into 10 subunits that are regarded as statistically independent.

The integrated $\mathrm{MC} / \mathrm{molecular}$ dynamic/Brownian dynamics simulation package MOLSIM for molecular systems was employed. ${ }^{16}$ The inclusion of the polarization energy lengthened the MC simulation times by the factor 2.5 , as compared to the time where the polarization energy evaluation was switched off.

\section{Systems}

Six types of systems are considered. They differ in terms (i) of the presence of either a stationary charge distribution or coions to be neutralized by the counterions, and (ii) of the volume available to the ions. Coions and oppositely charged counterions possess the absolute charge $\left|z_{\text {ion }}\right|=1$. Here, and in the following, co- and counterions are collectively referred to as ions. 
TABLE I. Specification of the types of systems considered, in terms of location of the stationary charge distributions and their counterions, and further specification in terms of the number of ions $N$, radius of region $1 R_{1}$, outer radius of region $2 R_{2}$, and values of the dielectric constants of medium $1 \varepsilon_{1}$, and medium $2 \varepsilon_{2} \cdot{ }^{a}$ The last column indicates the equation used to describe the reformulated Green's functions.

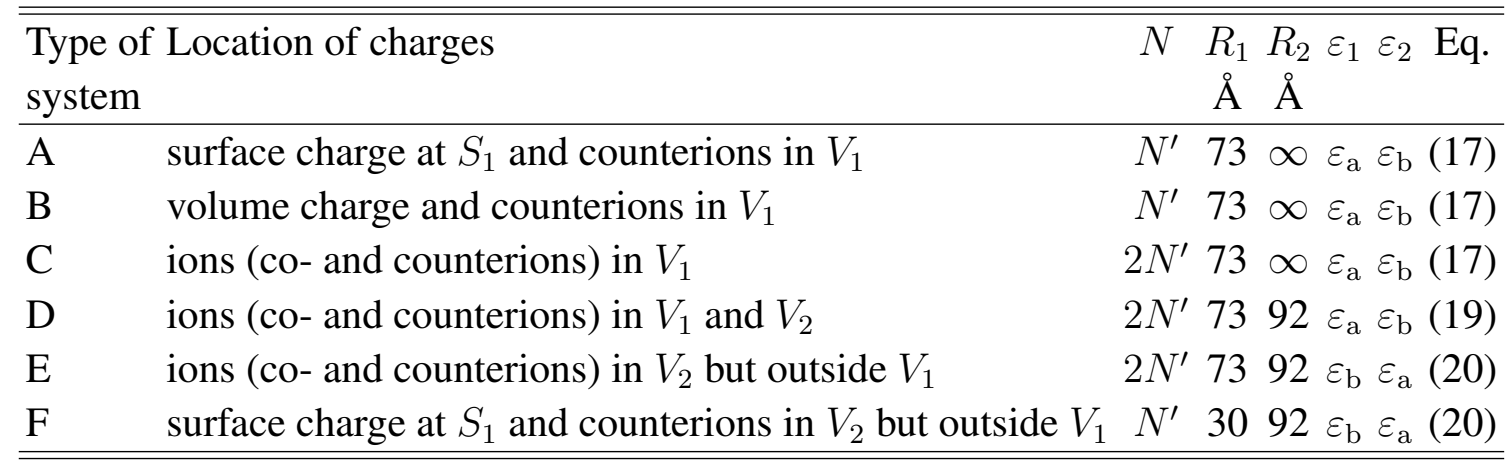

a a $N^{\prime} \in\{1,5,100\}, \varepsilon_{\mathrm{a}} \in\{80,40\}$, and $\varepsilon_{\mathrm{b}} \in\{80,40,20,10\}$. Other parameters: absolute ion charge $\left|z_{\text {ion }}\right|=1$ and temperature $T=298 \mathrm{~K}$.

The six type of systems constitute models for various experimental systems in soft matter such as microemulsions, micelles, reversed micelles, and cross-liked microgels.

The types of systems are divided into three groups according to the volume available for the ions. These groups matches the cases presented in Sec. II and are defined as follows:

1. Systems of type A-C comprise counterions confined in region 1, which neutralize (i) in systems of type A a uniform surface charge distribution at $S_{1}$, (ii) in systems of type B a uniform volume charge distribution in region 1 , and (iii) in systems of type $\mathrm{C}$ coions confined in region 1 .

2. Systems of type $\mathrm{D}$ have the ions confined in regions $1+2$ with $V_{1}=V_{2}$.

3. Systems of type E and F comprise counterions confined in region 2, which neutralize (i) in systems of type $\mathrm{E}$ coions confined in region 2 with $V_{2}=V_{1}$ and (ii) in systems of type $\mathrm{F}$ a uniform surface charge distribution at $S_{1}$ with $V_{2} \gg V_{1}$.

For each of the six types of systems, we consider throughout three different numbers of counterions $N^{\prime}$ combined with eight different choices of $\left(\varepsilon_{1}, \varepsilon_{2}\right)$ - a total of 144 systems. Table I provides a summary of the six types of systems and their specifications including values of $N^{\prime}$, $R_{1}, R_{2}, R_{\text {ion }}, \varepsilon_{1}, \varepsilon_{2}$, and temperature $T$; Fig. 2 displays the types schematically.

Instead of letting $\varepsilon_{1}$ and $\varepsilon_{2}$ refer to the dielectric constant of medium 1 and 2, respectively, we introduce $\varepsilon_{a}$ and $\varepsilon_{b}$ denoting the dielectric constant of the medium with ions and without ions, 
respectively. This can be made unambigously for all types of systems except those of type D, where ions appear in both regions - here we let $\varepsilon_{a}$ denote $\varepsilon_{1}$ and $\varepsilon_{b}$ denote $\varepsilon_{2}$. The reason for switching from $\left(\varepsilon_{1}, \varepsilon_{2}\right)$ to $\left(\varepsilon_{a}, \varepsilon_{b}\right)$ is to achieve a larger degree of similarity of the responses of the systems upon changing the dielectric constants.

\section{NUMERICAL RESULTS AND DISCUSSION}

This section is divided into two subsections: the first one contains results on the convergence of the expansion of the Green's function and the second one demonstrates effects of the dielectric discontinuities on structural properties. The two subsections are independent of each other and can be read in arbitrary order.

\section{A. Convergence}

Numerical results of our simulations are subject to (i) systematic errors arising from the truncation of the Green's function representing energetic contributions from the dielectric inhomogeneities given by Eqs. (17), (19), and (20) and (ii) statistical uncertainties due to the finite simulation length. Below, we examine how the average potential energy and the radial density distributions are affected by these two issues for systems containing $N^{\prime}=100$ counterions. Our focus will be on the systematic errors, and their magnitudes will be related to the statistical uncertainties.

\section{Potential energy}

The potential energy among the systems differs strongly, as the electrostatic interaction between counterions and balancing opposing charges is dependent of the volume available to the counterions as well as of the nature of the balancing charge and its space available. Moreover, the dielectric discontinuity is a source of further variation of the potential energy among the systems.

Figure 3 provides an overview of the statistical uncertainty of the potential energy $\delta U_{\text {stat }}$ as a function of $U_{l_{\max }=0}$, the latter denoting the average potential energy of the systems obtained with $l_{\max }=0$ [i.e., completely omitting the sum over $l$ in Eqs. (17), (19), and (20)]. Data for the

same type of system are given by same symbol. Symbols enclosed by circles represents results of homogeneous dielectric systems $\left(\varepsilon_{a}, \varepsilon_{b}\right)=(80,80)$. 
We observe that (i) the potential energy per ion varies from ca. -100 to $+100 \mathrm{~kJ} \mathrm{~mol}^{-1}$, (ii) the statistical uncertainty of most systems lies between $5 \times 10^{-4}$ to $10^{-2} \mathrm{~kJ} \mathrm{~mol}^{-1}$, and (iii) data of the different types of systems are clustered. In the case of homogeneous dielectric systems, systems of type A and F display the largest magnitude and the largest statistical uncertainty of the potential energy. In systems of type $A$, the repulsive Coulomb interaction among the counterions dominates, whereas in systems of type $\mathrm{F}$, the attractive surface charge-counterion interaction dominates. Generally, we see that the statistical uncertainty remains constant or increases with increasing difference of the dielectric constants of the two media. The increase in the statistical uncertainty is largest for systems of type D, most likely related to the existence of transitions of ions across the dielectric discontinuity.

We will now consider the effect of the truncation of the summation over $l$ in Eqs. (17), (19), and (20). We notice that for $\varepsilon_{2} / \varepsilon_{1} \rightarrow \infty, \delta G_{0}$ with $l_{\max }=0$ becomes exact. Thus, the infinite sum only contributes to the difference between the value of $\varepsilon_{2} / \varepsilon_{1}$ and $\infty$ (in fact, only a part of it). Initial simulations supported that the convergence of these expansions is very fast, and that it is sufficient to only examine the effect of including the first term $l=1$ of the infinite sum.

Figure 4 shows $\left|\delta U_{l}\right|$ with $\delta U_{l} \equiv U_{l_{\max }=1}-U_{l_{\max }=0}$ denoting the difference in the average potential energy emerging from simulations with $l_{\max }=1$ and $l_{\max }=0$ as a function of the statistical uncertainty of the average potential energy $\delta U_{\text {stat }}$, obtained with $l_{\max }=0$. We observe that the magnitude of the energy differences $\left|\delta U_{l}\right|$ in most cases is smaller or equal to the statistical uncertainty of the average potential energy $\delta U_{\text {stat }}$. Considering the length of these simulations providing small values of $\delta U_{\text {stat }}$, we conclude that the reformulation of the expansion given in Eq. (2) into Eqs. (17), (19), and (20) (i) leads to an expression possessing a very fast convergence and (ii) the expression of $\delta G_{0}$ obtained with $l_{\max }=0$ is sufficient to achieve an energy convergence of $0.01 \mathrm{~kJ} \mathrm{~mol}^{-1}$ or better. This holds for an extensive set of different type of systems at various combinations of dielectric constants.

The radial density distributions of mobile ions have been subjected to a similar analysis as the potential energy of the systems. Results of such an analysis applied to systems with the largest dielectric discontinuity $\left(\varepsilon_{a}, \varepsilon_{b}\right)=(80,10)$ of each type of system are presented in Fig. 5. In systems containing mobile co- and counterions, where they display same radial density distributions, only those of the counterions are shown. Here and in the following, we provide the reduced radial density distributions $n^{*}(r) \equiv n(r) /\langle n\rangle$, where $\langle n\rangle$ represents the average density in region 1 for systems of type $\mathrm{A}-\mathrm{C}$, in region $1+2$ for systems of type $\mathrm{D}$ and $\mathrm{F}$, and in region 2 for systems of 
type E.

First, we observe that the statistical uncertainty of the reduced radial density distribution $\delta n_{\text {stat }}^{*}(r)$ is largest at the origin and reduces continuously with increasing distance from the origin. This is a direct consequence of the spherical geometry and the use of sampling bins of equal length. The quantity $\delta n_{l}^{*}(r)$ represents the difference between the radial density distributions obtained from systems with the infinite sums in potential energy terms given in Eqs. (17), (19), and (20) truncated at $l_{\max }=1$ and 0 . Second and more importantly, we find $\left|\delta n_{l}^{*}(r)\right| \leq \delta n_{\text {stat }}^{*}(r)$ : the systematic error of truncating the infinite sum is bounded by the statistical uncertainty. Hence, we conclude that in our study the truncation of the infinite sums appearing in the potential energy expression and arising from the dielectric discontinuity causes a negligible effect on the radial density distributions of the mobile ions.

\section{B. Structural properties}

We will here provide radial density distributions of different systems illustrating the effect of a dielectric discontinuity. Such properties of some of the different types of systems have earlier been reported. ${ }^{8,11,12,14}$ Our departure will be homogeneous dielectric systems corresponding to water, for which we also will examine the role of different amounts of charge. In the following two subsections, we will present the effects of a dielectric inhomogeneity for the situations $\varepsilon_{a}>\varepsilon_{b}$ and $\varepsilon_{a}<\varepsilon_{b}$, respectively, limited to the largest charge selected and $N^{\prime}=100$ counterions.

In the following, systems of type $\mathrm{A}-\mathrm{C}$ will be discussed collectively. Whereas the representation of and volume available to the counterions are the same, we recall that these types of systems differ with respect to the appearance of the other charged species. In systems of type A, this charge is uniformly distributed at the surface of region 1, of type B it is uniformly distributed in the space of region 1 , and of type $\mathrm{C}$ it is divided into units carrying one elementary charge and are mobile within region 1 (see Fig. 2a-c). Hence, we can also assess how the different representation of one of the two charged species affects the radial density distribution of the counterions.

\section{Dielectric homogeneous systems with different amounts of charge}

Figure 6 provides reduced radial number density distributions of the counterions $n^{*}(r)$ for the six types of dielectric homogeneous systems at $\left(\varepsilon_{a}, \varepsilon_{b}\right)=(80,80)$. The distributions are given for 
different amount of charge and number of counterions, viz. $N^{\prime}=1,5$, and 100 .

Starting with $N^{\prime}=1$ counterion in the systems of type A, B, and C, we observe in Figs. 6a-c basically uniform counterion distributions. In all three types of systems, the relevant electrostatic interaction is smaller than the thermal energy and hence too small to significantly affect the properties of the systems. In more detail, in the system of type A the counterion distribution is truly uniform due to a constant external potential, cf. Eq. (22). Furthermore, in the system of type B the distribution is very weakly shifted toward the center of sphere 1 owing to the radially decreasing magnitude of the attractive external potential, cf. Eq. (24). In the system of type C, no external potential acts on the ions, nevertheless still a weak depletion of the ions appears near the surface of region 1.

Continuing with systems with increased charge and $N^{\prime}=5$ and 100 counterions, the relevant electrostatic interaction will progressively become more important relative to the thermal energy. The distribution of the counterions in systems of type A is for $N^{\prime}=5$ weakly and for $N^{\prime}=100$ markedly shifted toward the surface of region 1; in type B for $N^{\prime}=5$ depleted and for $N^{\prime}=100$ weakly accumulated at the surface of region 1; and in type $\mathrm{C}$ remains uniformly distributed but stronger depleted near the surface of region 1.

Thus, systems of type A-C display various responses upon an increase in charge. This variation originates from their different ability of establishing a local screening of ionic charge. In systems of type A, there is no such screening of the repulsion among the counterions, and their mutual repulsion forces them apart and toward the surface of region 1. In systems of type B with a uniform background charge, there are with $N^{\prime}>1$ two competing interactions. The mutual repulsion among the counterions is here counteracted by the the radial potential from the uniform volume charge density. With $N^{\prime}=100$ the balance has been altered such that the mutual repulsion among the counterions dominates over the attraction from the volume charge density. In systems of type $\mathrm{C}$, we have a local charge screening among ions of opposite charge, which acts on the length scale of the Debye screening length, here $\approx 10 \AA$ for $N^{\prime}=100$. Hence, the average ion density distribution becomes constant with the exception of a depletion near the surface of region 1. This depletion arises from the cohesive nature of the Coulomb interaction due to correlation effects in combination an anisotropic distribution of surrounding ions of an ion near a surface.

Three types of systems remain. Systems of type D and E possess coions occupying the same volume as the counterions, viz. $V_{1}+V_{2}$ in type $\mathrm{D}$ and $V_{2}$ in type $\mathrm{E}$, whereas in systems of type $\mathrm{F}$ the inner surface of region 2 possesses a uniform surface charge density. Figures $6 \mathrm{~d}-\mathrm{f}$ show the 
reduced radial density distributions for these three types of systems.

With $N^{\prime}=1$ counterion, the density distributions in the systems of type D and E are basically uniform; the depletion effect originating from correlation effects near the dielectric discontinuity is too small to be detected. In the system of type $\mathrm{F}$, a weak accumulation of the counterion at the charged surface appears. This is the expected outcome, given the attractive potential of the charged inner surface of region 2, cf. Eq. (22).

With increased charge and $N^{\prime}=5$ and 100 counterions, we observe a depletion of ions at the outer surface of region 2 in the systems of type D and E of the same correlation origin as in systems of type C. In systems of type D, (centers of) counterions are by construction absent in a shell with width of $4 \AA$. We notice that this gap is too narrow to affect the counterion distribution near it, whereas for systems of type $\mathrm{E}$ a depletion zone appears also at the inner surface of region 2. In the systems of type $F$, the accumulation of the counterions increases with increasing surface charge density. At the highest surface charge density and $N^{\prime}=100$ counerions, the contact value at $r=32 \AA$ amounts to $\approx 80$ and most counterions are located within a shell of $10 \AA$ from the inner surface.

Dielectric homogeneous systems at larger electrostatic coupling than that appearing in aqueous solutions of monovalent ions, e.g., realized by exchanging monovalent ions to multivalent ones or exchanging water to a solvent with a lower dielectric constant is also of interest. Figure 6 also displays selected reduced radial density distributions obtained at $\left(\varepsilon_{a}, \varepsilon_{b}\right)=(20,20)$ with $N^{\prime}=$ 100 and 50. These systems are trivially equivalent to those specified by $\left(z_{i o n}, \varepsilon_{a}, \varepsilon_{b}\right)=(2,80$, 80). The system with $N^{\prime}=100$ is related to the dielectric homogeneous system just discussed by replacing monovalent ions by divalent ones at constant ion number density and the one with $N^{\prime}$ $=50$ at constant ion charge density. For simplicity, these two systems are here referred to ones with divalent ions. Systems of type A, B, and F, remain charge neutral by having the charge of the counterions and the continuous charge distributions matching each other. In systems of type A and $\mathrm{F}$ with $N^{\prime}=100$ (dashed black curves), where all interactions are four-folded increased, we notice that the divalent counterions become more unevenly distributed than in the corresponding systems with monovalent counterions. The more uneven counterion distribution in system of type A is caused by a stronger counterion-counterion repulsion and in system of type $\mathrm{F}$ by a stronger surface-counterion attraction with a stronger, but not dominating, counterion-counterion repulsion. With $N^{\prime}=50$ (dotted black curves), where the charge of the continuous charge distribution remains the same as in the main study, obviously the increase in the accumulation of counterions 
becomes reduced. This reduced increase is in system of type A caused by the lower counterion number density and in system of type B by the weaker surface-counterion attraction, as compared to results with $N^{\prime}=100$. Finally, in systems of type B, C, D, and E, the main effect is an enhanced depletion of, now, divalent couterions in the system of type B and of divalent ions in systems of $\mathrm{C}, \mathrm{D}$, and $\mathrm{E}$ at the uncharged surfaces. The stronger ionic depletion originates from a larger loss of correlation energy near the surfaces as the cohesive nature of the Coulomb interaction increases with increasing ionic valence.

\section{Dielectric inhomogeneous systems: $\varepsilon_{a}>\varepsilon_{b}$}

We will now examine the effect of reducing the dielectric constant in the region free of mobil ions with systems of type $\mathrm{D}$ being the only exception. Results will be presented for $\varepsilon_{a}=80$ with $\varepsilon_{b}=80,40,20$, and 10 and with $N^{\prime}=100$ counterions. Table I gives an overview of all the systems investigated.

Figures $7 \mathrm{a}-\mathrm{c}$ display the evolution of the reduced radial density distributions in systems of types $\mathrm{A}-\mathrm{C}$ with decreasing $\varepsilon_{b}$. As to systems of type $\mathrm{A}-\mathrm{C}$, the major response to the decrease of the dielectric constant in medium 2 located outside medium 1 is a decrease in the counterion density in the vicinity of the dielectric discontinuities, otherwise the radial density distributions remain basically unaffected. The magnitude of the decrease of the distributions near the surfaces is similar across the three types of systems, and the decease becomes larger as the value of the dielectric constant in region 2 is decreased.

In systems of type D, a new feature of the ion distribution is shown in Fig. 6d. Here, the ions attain an equilibrium distribution between the two regions of different dielectric constant. To obtain a consistent description, an addition of Born ${ }^{1}$ terms have been made to the potential energy equations previously given. The Born energy comprises the free energy change of transferring an ion of a certain size from the gas phase to the fluid in question. From these Born energies, the unequal solubility of ions in media of different dielectric constants can be described and rationalized; a more negative Born term represents a higher solubility of an ion in a medium with a higher dielectric constant. Figure 7d indeed shows a lowering of the ion density in region 2 upon reducing $\varepsilon_{b}$. In addition to this strong response on the dielectric inhomogeneity, the ion distribution in regions 1 and 2 are affected by the dielectric discontinuity. The ion density near the discontinuity is in region 1 reduced and in region 2 increased with the largest effect appearing in the region with 
the largest dielectric constant.

The systems displaying the weakest response on the reduced dielectric content in region 1 are of type E (see Fig. 6e). On the level of the uncertainty of the current investigation, the ionic distribution is insensitive to the dielectric constant in region 1. As for the dielectric homogeneous system, at both the inner and outer surfaces of region 2 weak depletions appear.

Finally, we have systems of type F. Briefly, the reduction of the dielectric constant in region 1 reduces the accumulation of counterions at the charged interface as being manifested by a reduction of the maximum of the density distribution. The strength of this additional repulsion between a counterion and the dielectric discontinuity depends critically on the distance of the closest approach between the counterion and the discontinuity. This issues as well as the strong influence of the ionic charge on the repulsion have been subjects of previous studies. ${ }^{8,11,12,14}$

Hence, in five of the six types of systems, we find in addition to the effect of the Coulomb interaction that the ion density near the location of the dielectric discontinuities is affected by the discontinuity. The behavior is captured qualitatively by the conventional image charge picture, which is exact for a planar dielectric discontinuity: an ion experiences a repulsion from (attraction to) a dielectric discontinuity to a medium with a lower (higher) dielectric constant.

\section{Dielectric inhomogeneous systems: $\varepsilon_{a}<\varepsilon_{b}$}

Systems with dielectric inhomogeneities, where the ions reside in the medium of the lower dielectric constant, are experimentally difficult to realize. A barrier prohibiting the ions from leaving the medium with the lower dielectric constant and entering the medium with the higher dielectric constant becomes necessary. We will therefore only compare homogeneous systems with $\left(\varepsilon_{a}, \varepsilon_{b}\right)=(40,80)$ against inhomogeneous ones having $\left(\varepsilon_{a}, \varepsilon_{b}\right)=(40,80)$; again with $N^{\prime}=$ 100 counterions.

In systems of type A-C, Figs. 8a-c shows an enhanced counterion density near the surface of region 1 now surrounded by a region of higher dielectric constant. The spacial range of the increase is small, only ca. $5 \AA$. In the systems of type $\mathrm{C}$, closer inspection shows that the radial density distribution displays a minimum as a consequence of the different length scales of the correlation attraction and the surface polarization interaction.

The effect of the dielectric discontinuity on the radial ion density distribution of the system of type D with $\varepsilon_{a}<\varepsilon_{b}$ is basically reversed as compared to the case with $\varepsilon_{a}>\varepsilon_{b}$ (cf., Fig. 8d 
with Fig. 7d). The same applies to the counterion distribution of the system of type F, cf., Fig. $8 \mathrm{f}$ with Fig. 7f. Finally, in the system of type E, the depletion of ions at the surfaces of region 2 increases with the reduction of the dielectric constant of region 2. This is an example of the effect of increased Coulomb coupling. Moreover, the depletion at the two surfaces is unequal. At the inner surface, the depletion due to correlations is partly counteracted by the weaker attraction to the interface to region 1 with a higher dielectric constant.

\section{CONCLUSIONS}

We have derived and applied rapidly converged expressions of the Green's function of the Poisson equation for symmetric systems with spherical symmetric external potentials including a radial dielectric discontinuity. These expressions lengthen simulations with a single spherical dielectric discontinuity by only a factor of 2.5 , as compared to homogeneous dielectric systems. This will facilitate more accurate studies of models of dilute solutions of proteins, surfactant micelles, colloidal particles, etc., which generality possess a different dielectric constant as compared to the surrounding solution. The ability to include a single spherical dielectric discontinuity with only a moderate increase in computational cost makes it promising to simulate solutions of several particles having a dielectric constant different from the solvent. Such simulations restricted to two

particles have so far been made ${ }^{17}$ to establish two-particle potentials, whereas simulations with a larger number of particles are still computationally demanding.

The second outcome of our investigation is an ample number of examples where the competition between the mean-field Coulomb interaction, charge-surface polarization interaction, and correlation effects of the Coulomb interaction is illustrated. The effect of a dielectric discontinuity on the distribution of ions can conveniently be divided into two parts. First, in the presence of a spatial equilibrium of the ions across the discontinuity, the difference of the density of the ions in the two media is described in the present model by the Born energy. The parameters entering this expression are the ionic radius and the dielectric constants of the two media. Second, near the dielectric discontinuity, the surface polarization arising from the field of an nearby ion give rise to an repulsive (attractive) force acting on the ion at a discontinuity to a medium with a lower (higher) dielectric constant. Whereas the features of dielectric origin described here are well known, they are here discussed for a larger number of different systems and in the context of correlation effects. 


\section{ACKNOWLEDGMENTS}

Financial support by the Swedish Research Council (VR) through the Linnaeus grant Organizing Molecular Matter (OMM) center of excellence (239-2009-6794) and through individual grants to PL (2010-2253-78321-47) are gratefully acknowledged. Financial support by the BBSRC (BB/I017194/1) is gratefully acknowledged by LL.

\section{Appendix A: List of important variables and their symbols}

\begin{tabular}{lc}
\hline \hline Variable & Symbol \\
\hline number of ions of a given $z_{\text {ion }}$ & $N$ \\
volume of region 1 & $V_{1}$ \\
volume of region 2 & $V_{2}$ \\
surface of region 1 and inner surface of region 2 & $S_{1}$ \\
outer surface of region 2 & $S_{2}$ \\
radius of surface $S_{1}$ & $R_{1}$ \\
radius of surface $S_{2}$ & $R_{2}$ \\
relative dielectric constant of medium 1 & $\varepsilon_{1}$ \\
relative dielectric constant of medium 2 & $\varepsilon_{2}$ \\
radius of an ion & $R_{\text {ion }}$ \\
number of elementary charges of an ion & $z_{\text {ion }}$ \\
temperature & $T$ \\
upper end of summation over $l$ in Eqs. (17)-(20) & $l_{\max }$ \\
\hline \hline
\end{tabular}

Appendix B: Detailed derivations of Eqs. (17), (19), and (20)

In this appendix, we provide details of the derivation of the rapidly convergent expressions for the contribution to the Green's function from spherical dielectric inhomogeneities $\delta G_{0}\left(\mathbf{r}, \mathbf{r}^{\prime}\right)$. 


\section{Case 1:}

If both $\mathbf{r}$ and $\mathbf{r}^{\prime}$ lie inside the sphere, then from Eqs. (3) and (4)

$$
\begin{aligned}
\delta G_{0}\left(\mathbf{r}, \mathbf{r}^{\prime}\right) & =-\frac{1}{\varepsilon_{2} \eta R} \sum_{l=0}^{\infty} \frac{\Delta(l+1)}{l+\zeta} t^{l} P_{l}(\cos \gamma)+\frac{\eta^{-1}-1}{\varepsilon_{2}\left|\mathbf{r}-\mathbf{r}^{\prime}\right|} \\
& =-\frac{\Delta}{\varepsilon_{2} \eta R} \sum_{l=0}^{\infty}\left[1+\frac{1-\zeta}{l+\zeta}\right] t^{l} P_{l}(\cos \gamma)+\frac{\eta^{-1}-1}{\varepsilon_{2}\left|\mathbf{r}-\mathbf{r}^{\prime}\right|} \\
& =-\frac{\Delta}{\varepsilon_{2} \eta R}\left(1-2 t \cos \gamma+t^{2}\right)^{-1 / 2}-\frac{\Delta}{\varepsilon_{2} \eta R} \sum_{l=0}^{\infty} \frac{1-\zeta}{l+\zeta} t^{l} P_{l}(\cos \gamma)+\frac{\eta^{-1}-1}{\varepsilon_{2}\left|\mathbf{r}-\mathbf{r}^{\prime}\right|}
\end{aligned}
$$

where $t=r r^{\prime} / R^{2}$, and we have used the relation ${ }^{18}$ :

$$
\sum_{l=0}^{\infty} t^{l} P_{l}(x)=\left(1-2 x t+t^{2}\right)^{-1 / 2}
$$

If we define the Kelvin point as $\mathbf{r}_{K}=\left(R / r^{\prime}\right)^{2} \mathbf{r}^{\prime}$, then the first term in Eq. (B1) can be rewritten as:

$$
\left(1-2 t \cos \gamma+t^{2}\right)^{1 / 2}=\frac{1}{r_{K}}\left|\mathbf{r}-\mathbf{r}_{K}\right|
$$

The summation in the second term of Eq. (B1) can be rewritten as

$$
\begin{aligned}
\sum_{l=0}^{\infty} \frac{t^{l} P_{l}(\cos \gamma)}{l+\zeta} & =\sum_{l=0}^{\infty} \int_{0}^{t} d t^{\prime} t^{-\zeta} t^{\prime l+\zeta-1} P_{l}(\cos \gamma) \\
& =\int_{0}^{t} d t^{\prime} \frac{1}{t^{\prime}}\left(\frac{t^{\prime}}{t}\right)^{\zeta}\left(1-2 t^{\prime} \cos \gamma+t^{\prime 2}\right)^{-1 / 2} \\
& =\int_{r_{\mathrm{K}}}^{\infty} d x\left(\frac{r_{\mathrm{K}}}{x}\right)^{\zeta} \frac{1}{|\mathbf{r}-\mathbf{x}|}
\end{aligned}
$$

where $\mathbf{x}=\left(t / t^{\prime}\right) \mathbf{r}_{K}$. Therefore, we can rewrite the polarization contribution to the Green's function as

$$
\delta G_{0}\left(\mathbf{r}, \mathbf{r}^{\prime}\right)=-\frac{\Delta}{\varepsilon_{2} \eta} \frac{r_{\mathrm{K}} / R}{\left|\mathbf{r}-\mathbf{r}_{\mathrm{K}}\right|}-\frac{\Delta \zeta \eta}{\varepsilon_{2} \eta R} \int_{r_{\mathrm{K}}}^{\infty} d x\left(\frac{r_{\mathrm{K}}}{x}\right)^{\zeta} \frac{1}{|\mathbf{r}-\mathbf{x}|}+\frac{\eta^{-1}-1}{\varepsilon_{2}\left|\mathbf{r}-\mathbf{r}^{\prime}\right|}
$$


The summation in the second term in Eq. (B1) can also be written as

$$
\begin{aligned}
\sum_{l=0}^{\infty} \frac{t^{l} P_{l}(\cos \gamma)}{l+\zeta}= & \sum_{l=0}^{\infty}\left[\frac{1}{l+1}+\frac{1-\zeta}{(l+\zeta)(l+1)}\right] t^{l} P_{l}(\cos \gamma) \\
= & -\frac{1}{t} \ln \frac{\left(1-2 t \cos \gamma+t^{2}\right)^{1 / 2}-t+\cos \gamma}{1+\cos \gamma} \\
& +\eta\left[1+\sum_{l=1}^{\infty} \frac{\zeta}{(l+\zeta)(l+1)} t^{l} P_{l}(\cos \gamma)\right]
\end{aligned}
$$

where we have used the relation ${ }^{18}$ :

$$
\sum_{l=0}^{\infty} \frac{t^{l} P_{l}(x)}{l+1}=-\frac{1}{t} \ln \frac{\left(1-2 x t+t^{2}\right)^{1 / 2}-t+x}{1+x}
$$

Substituting Eq. (B2) into Eq. (B1) leads to Eq. (17).

\section{Case 2:}

This case is composed of two alternatives. If $\mathbf{r}$ lies outside the sphere and $\mathbf{r}^{\prime}$ lies inside, then we have

$$
\begin{aligned}
\delta G_{0}\left(\mathbf{r}, \mathbf{r}^{\prime}\right) & =\frac{1}{\varepsilon_{2} r} \sum_{l=0}^{\infty} \frac{\Delta l}{l+\zeta} t^{l} P_{l}(\cos \gamma) \\
& =\frac{\Delta}{\varepsilon_{2} r} \sum_{l=0}^{\infty}\left[1-\frac{\zeta}{l+\zeta}\right] t^{l} P_{l}(\cos \gamma) \\
& =\frac{\Delta}{\varepsilon_{2} r}\left(1-2 t \cos \gamma+t^{2}\right)^{-1 / 2}-\frac{\Delta \zeta}{\varepsilon_{2} r} \sum_{l=0}^{\infty} \frac{t^{l} P_{l}(\cos \gamma)}{l+\zeta}
\end{aligned}
$$

where $t=r^{\prime} / r$.

By defining the Kelvin point as $\mathbf{r}_{K}=\mathbf{r}^{\prime}$, we find

$$
\left(1-2 t \cos \gamma+t^{2}\right)^{1 / 2}=\frac{1}{r}\left|\mathbf{r}-\mathbf{r}_{K}\right|
$$


The summation in the second term can be recast as an integral:

$$
\begin{aligned}
\sum_{l=0}^{\infty} \frac{t^{l} P_{l}(\cos \gamma)}{l+\zeta} & =\sum_{l=0}^{\infty} \int_{0}^{t} d t^{\prime} t^{-\zeta} t^{\prime l+\zeta-1} P_{l}(\cos \gamma) \\
& =\frac{1}{t} \int_{0}^{t} d t^{\prime}\left(\frac{t}{t^{\prime}}\right)^{1-\zeta}\left(1-2 t^{\prime} \cos \gamma+t^{\prime 2}\right)^{-1 / 2} \\
& =\int_{0}^{r_{K}} d x\left(\frac{r_{K}}{x}\right)^{1-\zeta} \frac{1}{|\mathbf{r}-\mathbf{x}|}
\end{aligned}
$$

where $\mathbf{x}=\left(t^{\prime} / t\right) \mathbf{r}_{K}$. Substituting these two relations into Eq. (B3), we find Eq. (13).

Equation (19) can be derived for this case by simply substituting Eq. (B2) into Eq. (B3).

If $\mathbf{r}$ lies inside the sphere and $\mathbf{r}^{\prime}$ lies outside, then

$$
\delta G_{0}\left(\mathbf{r}, \mathbf{r}^{\prime}\right)=\frac{\Delta}{\varepsilon_{2} r^{\prime}}\left(1-2 t \cos \gamma+t^{2}\right)^{-1 / 2}-\frac{\Delta \zeta}{\varepsilon_{2} r^{\prime}} \sum_{l=0}^{\infty} \frac{t^{l} P_{l}(\cos \gamma)}{l+\zeta}
$$

where $t=r / r^{\prime}$.

Again, defining the Kelvin point as $\mathbf{r}_{K}=\mathbf{r}^{\prime}$, we find

$$
\left(1-2 t \cos \gamma+t^{2}\right)^{1 / 2}=\frac{1}{r^{\prime}}\left|\mathbf{r}-\mathbf{r}_{K}\right|
$$

In an idential manner to the previous case, the summation in the second term in Eq. (B4) can be rewritten as an integral. Substituting these two relations into Eq. (B3), we find Eq. (9).

Equation (19) can be derived for this case by simply substituting Eq. (B2) into Eq. (B4).

\section{Case 3:}

If both $\mathbf{r}$ and $\mathbf{r}^{\prime}$ lie outside the sphere, then we have

$$
\begin{aligned}
\delta G_{0}\left(\mathbf{r}, \mathbf{r}^{\prime}\right) & =\frac{1}{\varepsilon_{2} R} \sum_{l=0}^{\infty} \frac{\Delta l}{l+\zeta} t^{l+1} P_{l}(\cos \gamma) \\
& =\frac{\Delta t}{\varepsilon_{2} R} \sum_{l=0}^{\infty}\left[1-\frac{\zeta}{l+\zeta}\right] t^{l} P_{l}(\cos \gamma) \\
& =\frac{\Delta t}{\varepsilon_{2} R}\left(1-2 t \cos \gamma+t^{2}\right)^{-1 / 2}-\frac{\Delta \zeta t}{\varepsilon_{2} R} \sum_{l=0}^{\infty} \frac{t^{l} P_{l}(\cos \gamma)}{l+\zeta}
\end{aligned}
$$


where $t=R^{2} /\left(r r^{\prime}\right)$.

By defining the Kelvin point as $\mathbf{r}_{K}=\left(R / r^{\prime}\right)^{2} \mathbf{r}^{\prime}$, the first term can be rewritten, using the relation

$$
\left(1-2 t \cos \gamma+t^{2}\right)^{1 / 2}=\frac{1}{r}\left|\mathbf{r}-\mathbf{r}_{K}\right|
$$

The summation can be converted to an integral as

$$
\begin{aligned}
\sum_{l=0}^{\infty} \frac{t^{l} P_{l}(\cos \gamma)}{l+\zeta} & =\sum_{l=0}^{\infty} \int_{0}^{t} d t^{\prime} t^{-\zeta} t^{\prime l+\zeta-1} P_{l}(\cos \gamma) \\
& =\sum_{l=0}^{\infty} \int_{0}^{t} d t^{\prime}\left(\frac{t}{t^{\prime}}\right)^{1-\zeta} t^{\prime l} P_{l}(\cos \gamma) \\
& =\int_{0}^{t} d t^{\prime}\left(\frac{t}{t^{\prime}}\right)^{1-\zeta}\left(1-2 t^{\prime} \cos \gamma+t^{\prime 2}\right)^{-1 / 2} \\
& =\int_{0}^{r_{\mathrm{K}}} d x\left(\frac{r_{\mathrm{K}}}{x}\right)^{1-\zeta} \frac{1}{|\mathbf{r}-\mathbf{x}|}
\end{aligned}
$$

where $\mathbf{x}=\left(t^{\prime} / t\right) \mathbf{r}_{K}$.

Substituting the relation Eq. (B2) into Eq. (B5) leads directly to Eq. (20).

\section{REFERENCES}

${ }^{1}$ D. F. Evans and H. Wennerström, The Colloidal Domain where Physics, Chemistry, Biology, and Technology Meet, 2nd ed. (Wiley-VCH, New York, 1999).

${ }^{2}$ R. Messina, J. Phys.; Condens. Matter 21, 113102 (2009).

${ }^{3}$ C. J. F. Böttcher, Theory of Electric Polarization, Vol. 1 (Elsevier, Amsterdam, 1973).

${ }^{4}$ J. D. Jackson, Classical Electrodynamics (Wiley, New York, 1975).

${ }^{5}$ C. Tanford and J. G. Kirkwood, J. Am. Chem. Soc. 79, 5333 (1957).

${ }^{6}$ G. M. Torrie, J. P. Valleau, and G. N. Patey, J. Chem. Phys. 76, 4615 (1982).

${ }^{7}$ D. Bratko, B. Jönsson, and H. Wennerström, Chem. Phys. Letter 128, 449 (1986).

${ }^{8}$ P. Linse, J. Phys. Chem. 90, 6821 (1986).

${ }^{9}$ S. Levine and C. W. Outhwaite, J. Phys. Chem. 90, 6821 (1986).

${ }^{10}$ R. Kjellander and S. Marcelja, Chem. Phys. Lett. 142, 485 (1987).

${ }^{11}$ R. Messina, J. Chem. Phys. 117, 11062 (2002).

${ }^{12}$ A. P. dos Santos, A. Bakhshandeh, and Y. Levin, J. Chem. Phys. 135, 044124 (2011). 
${ }^{13}$ I. V. Lindell, Radios Sci. 27, 1 (2002).

${ }^{14}$ L. Lue and P. Linse, J. Chem. Phys. 135, 224508 (2011).

${ }^{15}$ R. A. Curtis and L. Lue, J. Chem. Phys. 123, 174702 (2005).

${ }^{16}$ P. Linse, MOLSIM, Version 5.0, Lund University, Sweden (2011).

${ }^{17}$ J. Reščič and P. Linse, J. Chem. Phys. 129, 114505 (2008).

${ }^{18}$ G. Arfken, Mathematical Methods for Physicists, 3rd ed. (Academic Press, San Diego, 1985). 

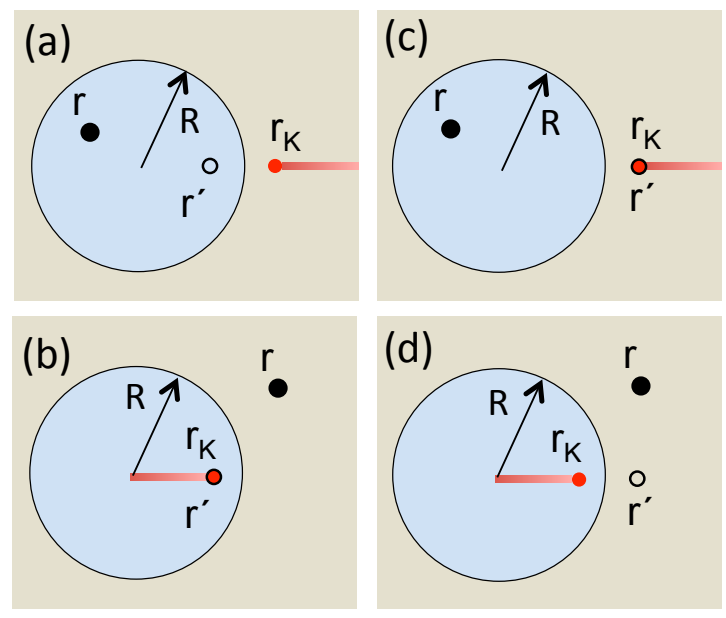

FIG. 1. A spherical dielectric discontinuity with radius $\mathbf{R}$ between two media with a unit point charge at $\mathbf{r}^{\prime}$ (open circles) localized [(a) and (b)] inside and [(c) and (d)] outside the discontinuity and the position $\mathbf{r}$ (solid black circles) of the generated potential located [(a) and (c)] inside and [(b) and (d)] outside the discontinuity. The potential at $\mathbf{r}$ can be viewed as being generated by an image point charge at $\mathbf{r}_{\mathrm{K}}$ (red circles) and an image line charge of variable charge density (red lines), which sign, magnitude, and location depend on the position of $\mathbf{r}^{\prime}$ and $\mathbf{r}$. 

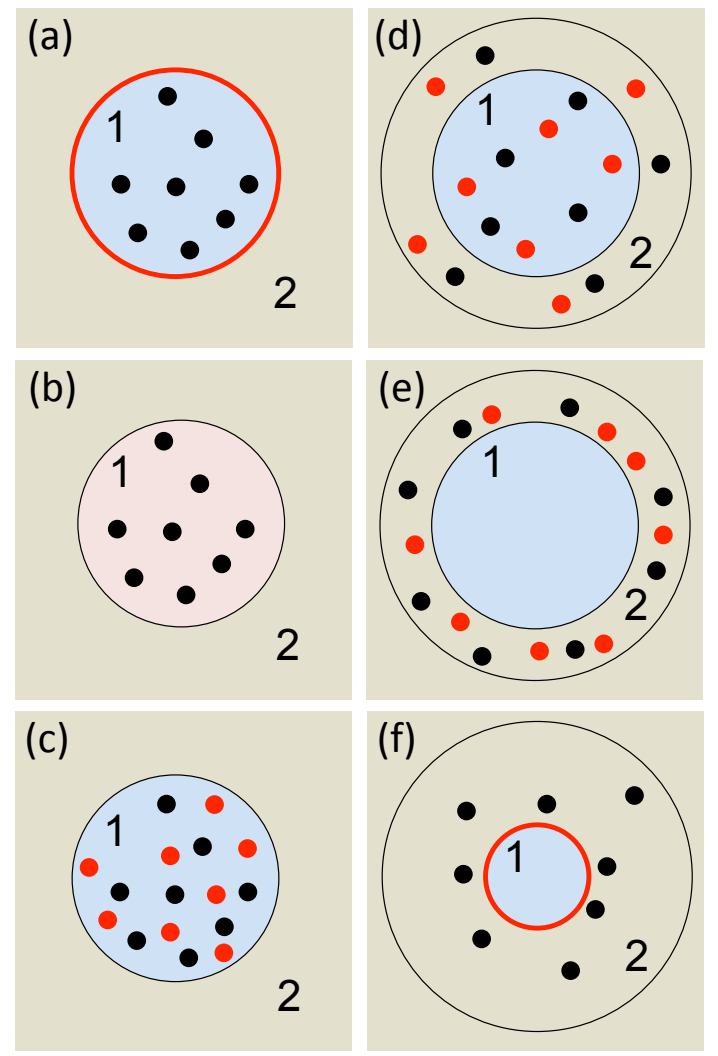

FIG. 2. Overview of different types of systems. Systems of all types contain a spherical region of medium 1 with dielectric constant $\varepsilon_{1}$ surrounded by medium 2 of infinite size with dielectric constant $\varepsilon_{2}$. In the types of systems given in (d), (e), and (f) a second sphere is present and divides medium 2 into two parts. Furthermore, in (a) region 1 possesses a uniform surface charge density and counterions are confined within the sphere, in (b) region 1 possesses a uniform volume charge density and counterions are confined within the sphere, in (c) ions are confined within region 1, in (d) ions are confined within region $1+2$, in (e) ions are confined within region 2 , and in (f) region 1 possesses a uniform surface charge density and counterions are confined in region 2. 


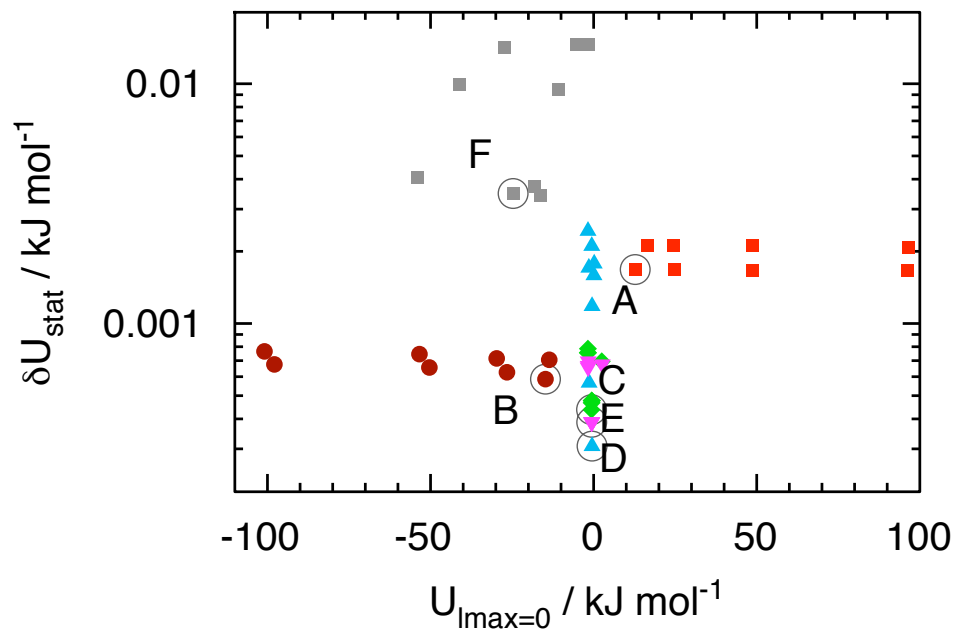

FIG. 3. Statistical uncertainty of the potential energy, $\delta U_{\text {stat }}$, arising from simulations of finite length, as a function of the potential energy evaluated with $l_{\max }=0, U_{l_{\max }=0}$, in a lin-log scale for systems of type A (squares), B (circles), C (diamonds), D (triangles), E (nablas), and F (squares) obtained with $N^{\prime}=100$ counterions at various values of $\left(\varepsilon_{a}, \varepsilon_{b}\right)$. Data for $\left(\varepsilon_{a}, \varepsilon_{b}\right)=(80,80)$ are enclosed by circles. 


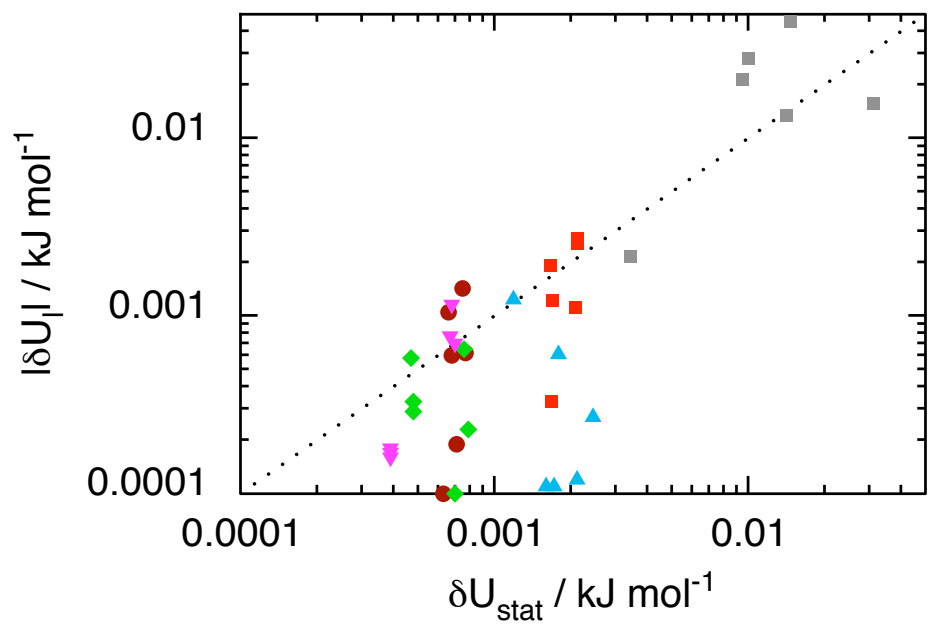

FIG. 4. Absolute difference of potential energy, $\left|\delta U_{l}\right| \equiv\left|U_{l_{\max }=1}-U_{l_{\max }=0}\right|$, as a function of the statistical uncertainty $\delta U_{\text {stat }}$ in a log-log scale for systems of type A (squares), B (circles), C (diamonds), D (triangles), $\mathrm{E}$ (nablas), and $\mathrm{F}$ (squares) obtained with $N^{\prime}=100$ counterions and $l_{\max }=0$. Symbols of a given type represents the variation obtained by the subset of six combinations of $\left(\varepsilon_{1}, \varepsilon_{2}\right)$ providing inhomogeneous systems. The diagonal represents the condition $\delta U_{\text {stat }}=\left|\delta U_{l}\right|$. 


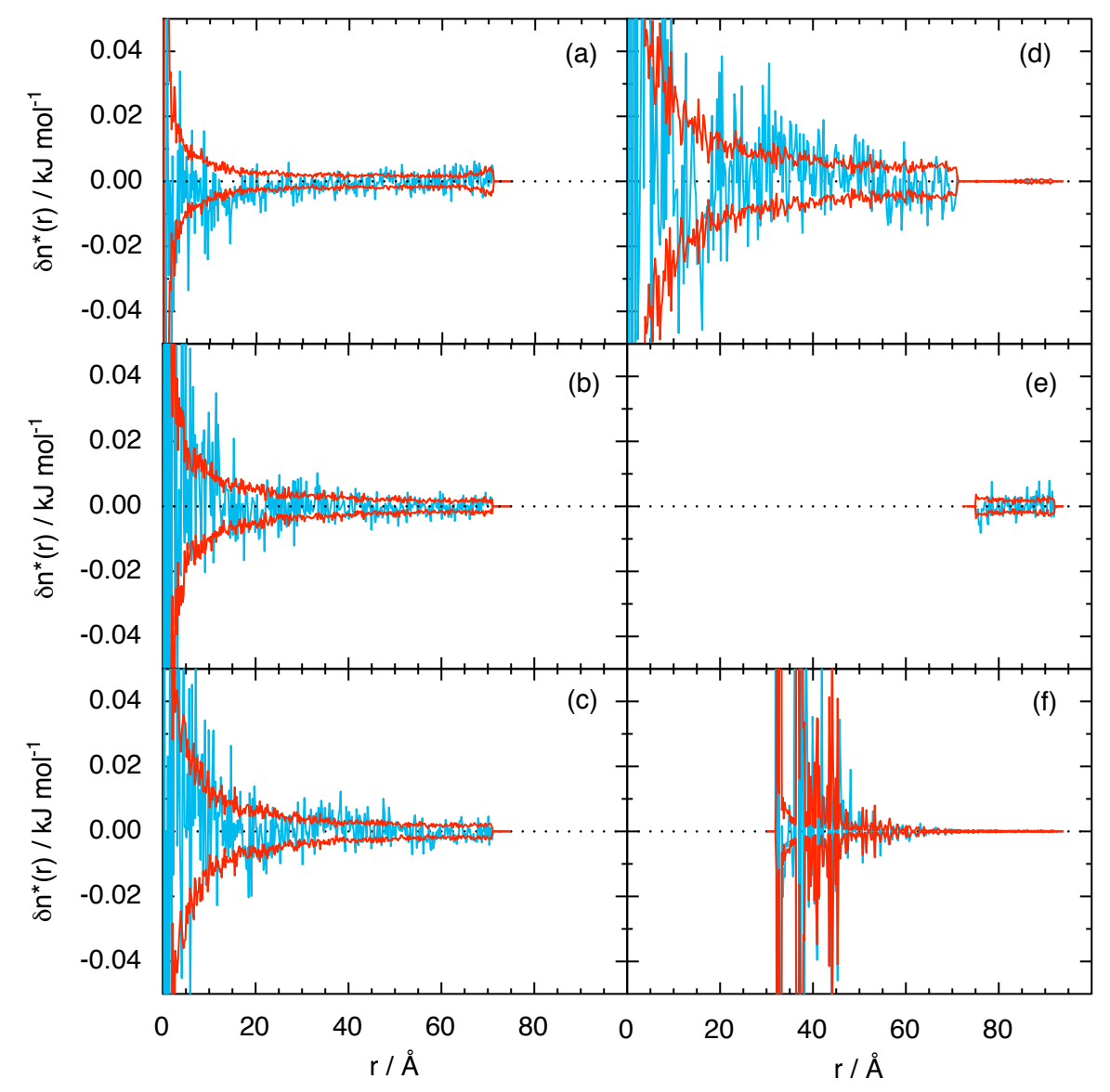

FIG. 5. Difference of the reduced radial density distribution $\delta n_{l}^{*}(r)$ from simulations with $l_{\max }=0$ and 1 entering in Eqs. (17), (19), and (20) (blue curves) and the statistical uncertainty of the reduced radial density distribution $\delta n_{\text {stat }}^{*}(r)$ given as \pm one standard deviation (red curves) for systems of type (a) A , (b) B, (c) C, (d) D, (e) E, and (f) F obtained with $N^{\prime}=100$ counterions and $\left(\varepsilon_{a}, \varepsilon_{b}\right)=(80,10)$. In (f) the scale of the ordinate is 10 times as large as those in the other panels. 




FIG. 6. Reduced radial density distributions of the counterions $n^{*}(r)$ vs radial distance $r$ for systems of type (a) A , (b) B, (c) C, (d) D, (e) E, and (f) F for $N^{\prime}=1$ (blue), 5 (green), and 100 (red) counterions at $\left(\varepsilon_{a}, \varepsilon_{b}\right)=(80,80)$ and for $N^{\prime}=100$ (black dashed curves) and $N^{\prime}=50$ (black dotted curves) counterions at $\left(\varepsilon_{a}, \varepsilon_{b}\right)=(20,20)$ with selected contact values indicated. Dotted lines represent the distribution $n^{*}(r)=$ 1. The upper limit of the ordinate is 6 in panel (a), 3 in panels (b), (c), (d), and (e), and 6 in panel (f). 




FIG. 7. Reduced radial density distributions of the counterions $n^{*}(r)$ vs radial distance $r$ for systems of type (a) A, (b) B, (c) C, (d) D, (e) E, and (f) F for $N^{\prime}=100$ counterions at $\varepsilon_{a}=80$ and $\varepsilon_{b}=80$ (red), 40 (green), 20 (blue), and 10 (black) with selected contact values indicated. Dotted lines represent the distribution $n^{*}(r)=1$. The upper limit of the ordinate is 6 in panel (a), 3 in panels (b), (c), (d), and (e), and 100 in panel (f). 


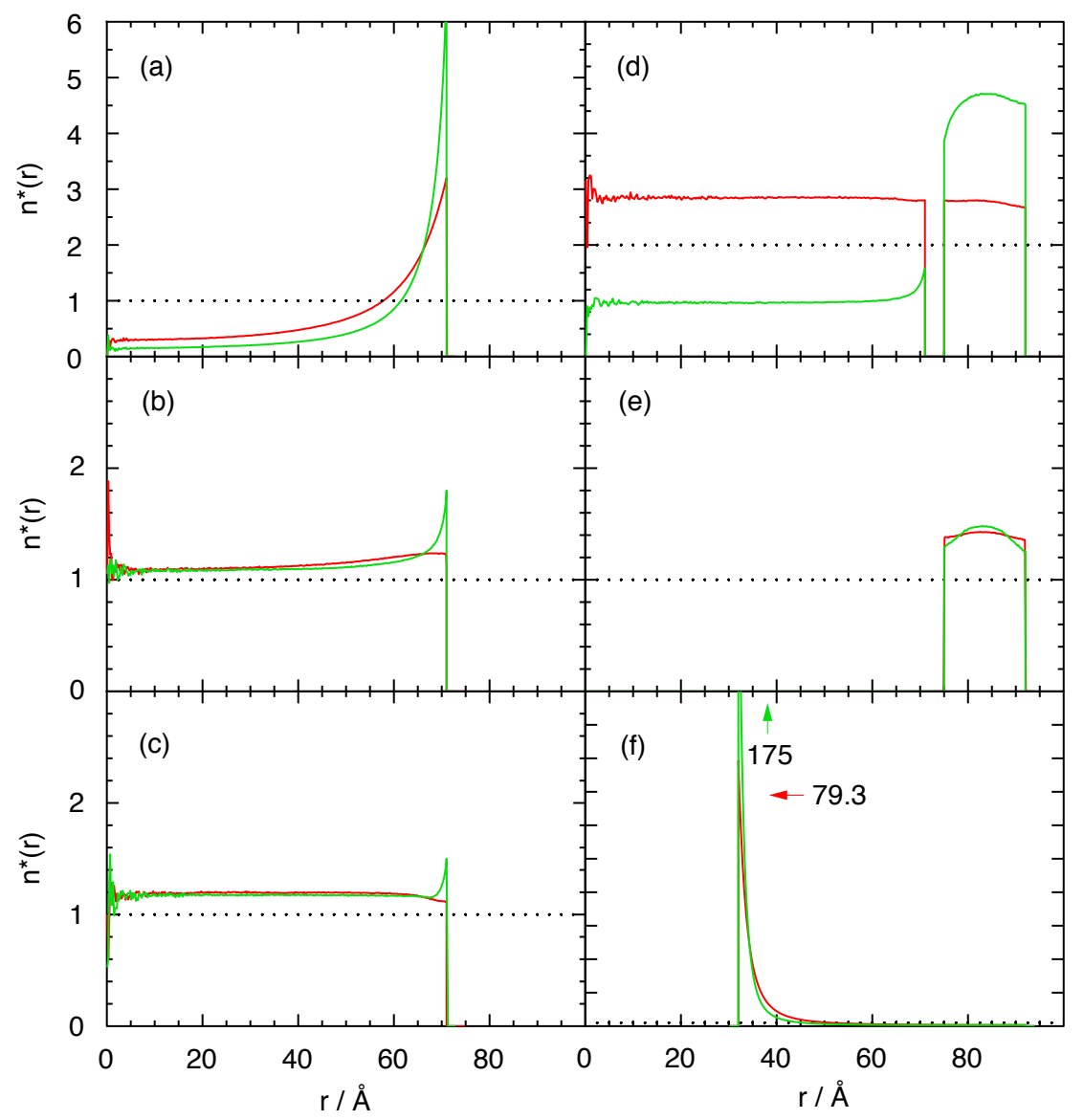

FIG. 8. Reduced radial density distributions of the counterions $n *(r)$ vs radial distance $r$ for systems of type (a) A, (b) B, (c) C, (d) D, (e) E, and (f) F for $N^{\prime}=100$ counterions at $\varepsilon_{b}=80$ and $\varepsilon_{a}=80$ (red) and 40 (green) with selected contact values indicated. Dotted lines represent the distribution $n^{*}(r)=1$. The upper limit of the ordinate is 6 in panel (a), 3 in panel (b), (c), (d), and (e), and 100 in panel (f). 